\title{
The Detection of Dark Galaxies in Blind HI Surveys
}

\author{
Jonathan I. Davies
}

School of Physics and Astronomy, Cardiff University, Queen's Buildings, 5 The Parade, Cardiff CF24 3YB, Wales, UK.

\begin{abstract}
One explanation for the disparity between Cold Dark Matter predictions of galaxy numbers and observations could be that there are numerous dark galaxies in the Universe. These galaxies may still contain baryons, but no stars, and may be detectable in the $21 \mathrm{~cm}$ line of atomic hydrogen. In this paper we describe a numerical model of the galaxy population and predict what might be found in blind $21 \mathrm{~cm}$ surveys. We describe the detection of a dark galaxy candidate (VIRGOHI21) and discuss a model of its origin.
\end{abstract}

Keywords. Galaxies: formation, interactions, Cosmology: dark matter

\section{Introduction}

The currently favoured Cold Dark Matter (CDM) model of galaxy formation predicts many more Dark Matter (DM) halos than are observable as galaxies. Amongst other speculations the existence of dark galaxies offers one solution (Verde et al., 2002). It is possible that the currently observable galaxies are just some fraction of a larger and more varied population of DM halos. The exsistence of objects that contain HI, but no stars, has been known for many years - for example High Velocity Clouds, tidal tails and clouds close to optically bright galaxies, but none of these have the characteristics that might lead them to be described as galaxies. To count as a dark galaxy a source requires HI emission over galactic scales (greater than a few $\mathrm{kpc}$ ) with velocity widths and profiles commensurate with a dynamically stable system (velocities greater than a few tens of $\mathrm{km} \mathrm{s}^{-1}$ ).

In this paper we first of all want to re-address the issue of whether dark galaxies can exist within our current models of galaxy formation and particularly examine the recent contention by Taylor and Webster (2005) (see also Taylor these proceedings) that almost all galaxies containing HI will form stars. Secondly we want to discuss whether the existence of dark galaxies is compatable with the results of previous blind HI surveys. Thirdly we will discuss the dark galaxy candidate VIRGOHI21 found by us in a previous blind HI survey and fourthly we will describe a numerical model of the observations we have made of VIRGOHI21.

\section{The model}

We start by modelling the galaxy population. We have used the prescription of Mo et al., (1998) [MMW] to model thin self-gravitating baryonic galactic discs within NFW (Navarro, Frenk and White, 1995,1996) dark matter halos. The model is fully described in Davies et al., (2006). Below we just give the 'recipe' for the simulated galaxies We select a DM halo mass (M) for each galaxy simulated from a Schechter function.

$$
\Theta(M) d M=\left(M / M^{*}\right)^{-\alpha} \exp -\left(M / M^{*}\right) d\left(M / M^{*}\right)
$$


with $M^{*}=10^{12} M_{\odot}$, low mass slope $\alpha=-1.5$ and mass range $10^{8}-10^{13} M_{\odot}$ (Dalcanton et al. 1997). Each halo has a NFW profile concentration index $\mathrm{C}=10$ (MMW). A fraction $m_{d}$ of the mass of each halo collapses to form a baryonic disc embedded in the halo. The fraction of the total angular momentum associated with the disc $\left(j_{d}\right)$ is the same as the fraction of the mass associated with the disc $\left(j_{d}=m_{d}\right)(\mathrm{MMW})$. The galaxy spin parameter $(\lambda)$ is taken from the lognormal distribution

$$
p(\lambda) d \lambda=\frac{1}{\sigma_{\lambda} \sqrt{2 \pi}} \exp \frac{\ln ^{2}(\lambda /<\lambda>)}{2 \sigma_{\lambda}^{2}} \frac{d \lambda}{\lambda}
$$

where $\langle\lambda\rangle=0.05$ and $\sigma_{\lambda}=0.5$ (Warren et al., 1992). As in MMW we assume that our galaxies form at $z=0(H(z)=1.0)$ and that $h=0.72\left(H_{o}=72 \mathrm{~km} \mathrm{~s}^{-1} \mathrm{Mpc}^{-1}\right)$. We calculate the maximum circular velocity $V_{c}$ in $\mathrm{km} \mathrm{s}^{-1}$ of each disc using

$$
V_{c}=V_{200} f_{V}
$$

where

$$
V_{200}=0.017(M H(z) h)^{1 / 3}
$$

and $f_{V}$ is a dimensionless factor obtained from the fitting function given in MMW (accurate to $\approx 20 \%)$. The exponential scale length of each disc $\left(R_{d}\right.$ in $\left.\mathrm{kpc}\right)$ is calculated using

$$
R_{d}=R_{d}^{i s o} f_{c}^{-0.5} f_{R}
$$

where

$$
R_{d}^{i s o}=0.7(H(z) h)^{-1.0} \lambda V_{200}\left(j_{d} / m_{d}\right)
$$

and $f_{c}$ (accurate to $\approx 1 \%$ ) and $f_{R}$ (accurate to $\approx 20 \%$ ) are again dimensionless fitting functions given in MMW. We can also calculate a disc central gas column density in $\mathrm{cm}^{-2}$ (assuming at this point that we have a gas disc) using

$$
\Sigma_{0}=\frac{2.0 \times 10^{13} m_{d} M}{R_{d}^{2}}
$$

The above model produces discs with a wide range of properties, but not all of them will be stable. Following MMW we use a disc stability criterion $(e)$ to assess the stability of each disc (see Efstathiou et al. 1982). Only discs with $e>1.0$ will be included in our final list of galaxies.

$$
e=\frac{1}{2^{1 / 4}}\left(\lambda^{\prime} / m_{d}\right)^{0.5} f_{c}^{-1 / 4} f_{R}^{1 / 2} f_{V}
$$

where $\lambda^{\prime}=\lambda j_{d} / m_{d}$.

The above model can be used to produce a simulated sample of stable gaseous discs embedded within DM halos. For each halo we have a total mass $(\mathrm{M})$, disc mass $\left(m_{d} M\right)$, disc circular velocity $\left(V_{c}\right)$, exponential disc scale length $\left(R_{d}\right)$ and central gas column density $\left(\Sigma_{0}\right)$. We can distinguish within our simulation between galaxy discs that can form stars $\left(\Sigma_{0}>5 \times 10^{20} \mathrm{~cm}^{-2}\right)$ and those that cannot $\left(\Sigma_{0}<5 \times 10^{20} \mathrm{~cm}^{-2}\right)$ (Schaye, 2004). $m_{d}$ turns out to be the crucial parameter when deciding whether dark galaxies are prolific within the above galaxy formation scenario. For a small value of $m_{d}$ many low column density discs are formed that are not able to form stars - dark galaxies (see fig. 1). Mo et al., (1998) conclude, after comparing their model with observations, that $m_{d}<0.05$ for typical disc galaxies. For the purpose of our simulation we will conservatively choose values of $m_{d}$ from a random uniform distribution between 0.01 and 0.05. There is also the possiblity that the hydrogen in dark galaxies is ionised by the extragalactic UV background. Theoretical models predict ionisation of atomic hydrogen 


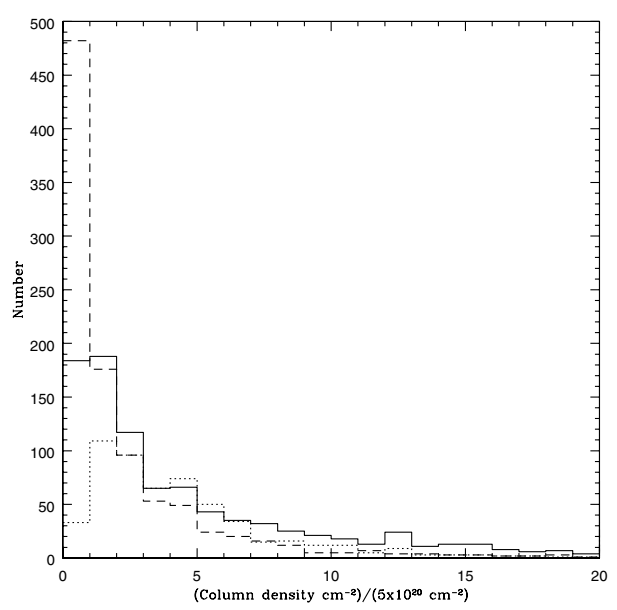

Figure 1. The distribution of column densities (normalised by $\Sigma_{C r i t}=5 \times 10^{20} \mathrm{~cm}^{-2}$ ) for three values of $m_{d}$. Large dash $m_{d}=0.01$, solid line $m_{d}=0.025$, small dash $m_{d}=0.05$. Only objects in the first column on the left will remain as dark galaxies. We see that small values of $m_{d}$ lead to relatively large numbers of dark galaxies.

layers that have column densities of a few $\times 10^{19} \mathrm{~cm}^{-2}$ (Maloney 1993). Thus there may be a narrow range of column densities approximately between $5 \times 10^{19}$ and $5 \times 10^{20} \mathrm{~cm}^{-2}$ over which dark galaxies can exist and be detectable by $21 \mathrm{~cm}$ surveys.

The prediction from the model is that about $80 \%$ of stable discs have sufficiently high HI column densities to form stars. If all discs were still gaseous then about $2 \%$ of the gas would be in dark galaxies. Given that those galaxies able to form stars will have done so we can adjust for the fraction of gas now turned into stars by dividing by $\left(1+\frac{1}{M_{H I} / L_{B}}\right)$. Assuming that $L_{B}$ relates directly to stellar mass and taking the Milky Way value of $M_{H I} / L_{B} \approx 0.1$ the model then predicts that $\approx 15 \%$ of the current $\mathrm{HI}$ in the Universe resides in dark galaxies.

\section{Dark galaxies in previous blind HI surveys}

To date the most complete large area $21 \mathrm{~cm}$ blind survey is HIPASS and the catalogue of sources is known as HICAT. Our simulation predicts that just $0.3 \%$ of the total HI detected in HICAT is in the form of dark galaxies. This compares with $15 \%$ within the total simulated galaxy population i.e. we predict that many dark galaxies will go undetected by HICAT. Normalising the total numbers of galaxies in the simulation to the number in HICAT we predict that there should be $\approx 80$ dark galaxies in HICAT. In Fig. 2 we show histograms of the HI mass and velocity widths for the dark galaxies in the simulation. It is clear that the majority of dark galaxies are predicted to have low HI masses and narrow velocity widths.

Recently Doyle et al. (2005) carried out a search for optical companions of the $4315 \mathrm{HI}$ sources in the HICAT. Their conclusion was that there are no 'isolated' dark galaxies. The statistics of what was found is as follows: there are optical counterparts for $3618 \mathrm{HI}$ sources (84\%), this includes what they describe as 'good guesses', 972 of these $(23 \%)$ are described as 'multiple possible matches', 2512 (58\%) have confirmed optical velocities, $216(5 \%)$ have no obvious optical galaxies present (most of these are in crowded fields, along the Galactic plane or have high extinction). They also say that some of their optical 

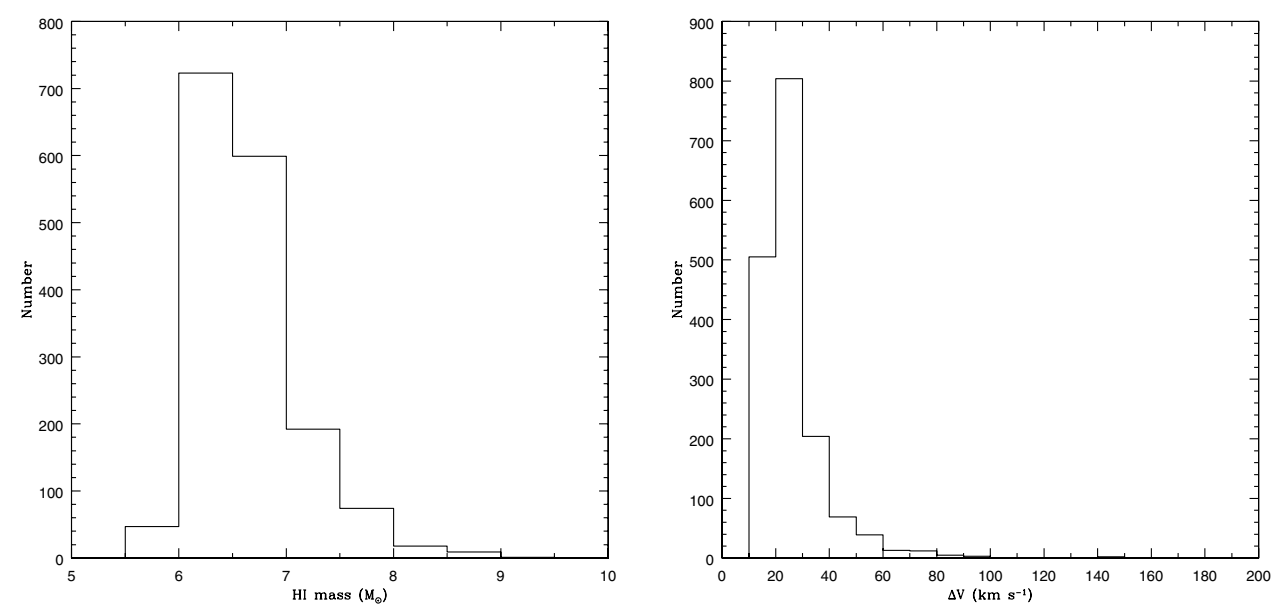

Figure 2. The simulation prediction for the distribution HI masses (left) and velocity widths (right) for dark galaxies.

\begin{tabular}{l|c|c|c} 
Survey & $\begin{array}{c}\text { \% Predicted } \\
\text { Dark Galaxies }\end{array}$ & $\begin{array}{c}\text { Total HI } \\
\text { Detected }\end{array}$ & $\begin{array}{c}\text { Predicted Number of } \\
\text { Dark galaxies in Survey }\end{array}$ \\
\hline HIPASS & 2 & 4315 & 80 \\
ALFALFA & 4 & 20000 & 800 \\
VIRGOHI & 6 & 31 & 2 \\
AGES & 23 & - & -
\end{tabular}

Table 1. The predicted percentage of dark galaxies in various blind HI surveys. The 20,000 detections for ALFALFA is a prediction from initial observations. The numbers for the VIRGOHI and AGES surveys are for a galaxy population simulated at about the distance of the Virgo cluster $(16 \mathrm{Mpc})$. As AGES has only just started we can only give the expected percentage.

matches disagree with the optical matches given in the HIPASS bright galaxy catalogue (Koribalski et al. 2004). Doyle et al. define a dark galaxy '..as any HI source that contains gas (and dark matter) but no detectable stars, and is sufficiently far away from other galaxies, groups or clusters such that a tidal origin can be excluded'. It is also important to note that the Parkes beam is about 14 arc min across and that Doyle et al. assume a 'velocity match' if HI and optical velocities agree to $400 \mathrm{~km} \mathrm{~s}^{-1}$. We do not believe that the Doyle et al. (2005) identification statistics and selection criteria rule out our predicted 80 dark galaxies within HICAT.

In Table 1 we give the predictions for the numbers of dark galaxies in various other surveys, ALFALFA (see Giovanelli these proceedings), AGES (see Minchin these proceedings) and VIRGOHI (see below).

\section{VIRGOHI21 a dark galaxy candidate}

In 2004 we used the Jodrell Bank multi-beam instrument to carry out a deep survey of part of the Virgo cluster (Davies et al., 2004). The Virgo survey (VIRGOHI) reduced the 

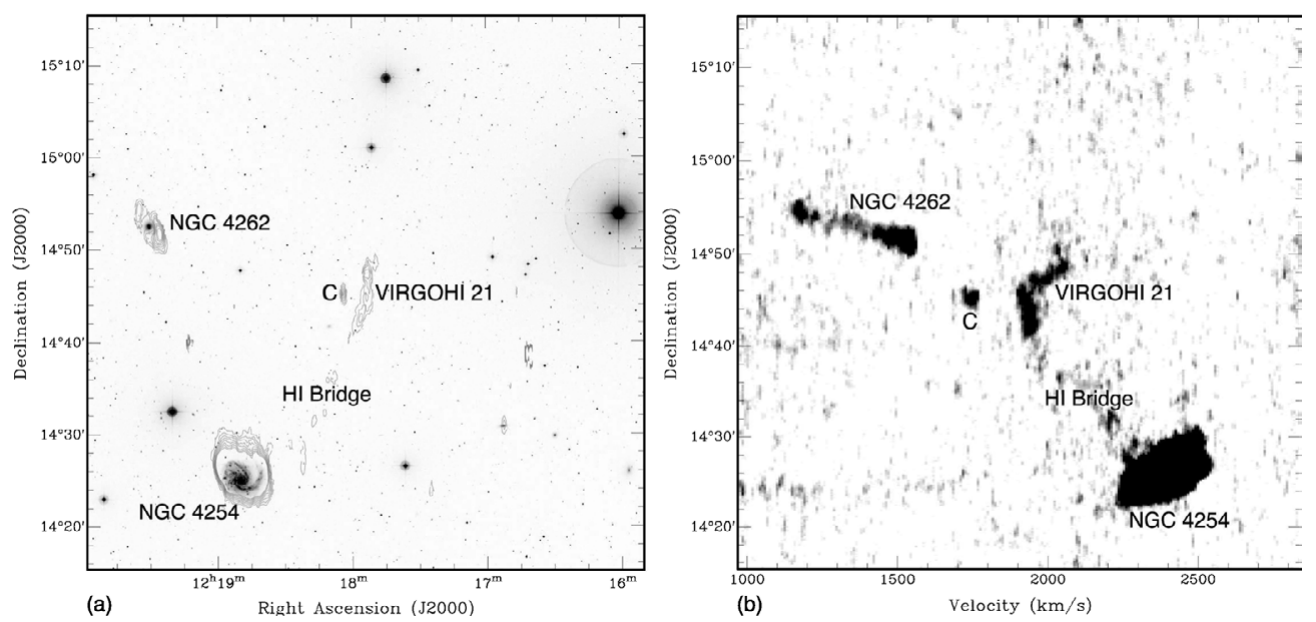

Figure 3. Westerbork observations of VIRGOHI21 and its immediate surroundings (reproduced from Minchin et al., 2007). (a) HI contour map of the 21-cm observations, superimposed on a 1 square degree negative DSS image. Contours are from $2.5 \times 10^{19}$ to $2.5 \times 10^{20} \mathrm{~cm}^{-2}$ at intervals of $2.5 \times 10^{19} \mathrm{~cm}^{-2}$. (b) Declination-velocity projection of the HI data cube.

$\approx 14$ mJy per channel of the HIJASS survey to $\approx 4$ mJy enabling us to look for both lower mass and lower column density hydrogen than would be detected in the normal survey. The area observed was also one in which we know there is a large number of nearby optically detected galaxies. Davies et al. identified four objects that had HI detections but no obvious optical counterparts. One of these was subsequently rejected as noise, a second was later identified, after obtaining new deep CCD data, with a very faint dwarf galaxy. The third object has subsequently been associated with debris stripped from NGC4388 (Oosterloo and van Gorkom, 2005). The fourth object, VIRGOHI21, remains as a candidate for a dark galaxy (Minchin et al. 2005, Minchin et al. 2007). VIRGOHI21 is extended over a region of about $10 \mathrm{kpc}$ and has a $\mathrm{HI}$ velocity width of about $200 \mathrm{~km} \mathrm{~s}^{-1}$ according to the HI data it is a typical galaxy, but there is no optical counterpart down to very low surface brightness limits $(\approx 33 B \mu$ from HST data, Minchin et al., 2007).

Fig. 3a shows a neutral hydrogen HI contour map of the field superimposed on a negative optical image. VIRGOHI21 is the elongated structure in the center (which is at about $2000 \mathrm{~km} \mathrm{~s}^{-1}$ ). A faint bridge can be seen stretching down to the prominent spiral NGC4254 (2400 $\left.\mathrm{km} \mathrm{s}^{-1}\right)$ while the other two sources, NGC4262 (1350 km s${ }^{-1}$, upper left) and the faint galaxy ' $\mathrm{C}$ ' $\left(1750 \mathrm{~km} \mathrm{~s}^{-1}\right.$, immediately to the left of VIRGOHI21) appear unconnected. Fig. 3b shows the velocity-declination projection of the full 3 dimensional data cube. The bridge between VIRGOHI21 and NGC 4254 is clear and obvious, as is the lack of any connection between VIRGOHI21 and either NGC4262 or galaxy C. The most interesting feature is the apparent very sharpe change in velocity along the bridge. If this is the position where the material in the bridge links to that in a rotating disc (VIRGOHI21) then the disc has the properties given in Table 2.

The very low surface-brightness limits give an upper limit to the luminosity of the disk of $1.9 \times 10^{5} L_{\odot}$, giving a lower limit for $M_{d y n} / L_{B}$ of at least $10^{6} M_{\odot} / L_{\odot}$, whereas normal galaxies have $<50$. 
Table 2. Properties of the putative dark disk

\begin{tabular}{ll}
\hline Diameter $2 R,\left[\right.$ from $+14^{\circ} 46^{\prime}$ to $\left.49^{\prime}\right]$ & $14 \mathrm{kpc}$ \\
Circular Velocity $V_{c},[(2100-1900) / 2]$ & $100 \mathrm{~km} \mathrm{~s}^{-1}$ \\
Spin Period $P,\left[2 \pi R / V_{c}\right]$ & $4 \times 10^{8}$ years \\
Total Mass $M_{T},\left[R V_{c}^{2} / G\right]$ & $2 \times 10^{10} M_{\odot}$ \\
Hydrogen Mass (disc) $M_{H I},\left[F_{H I}=0.6 \mathrm{Jy} \mathrm{km} \mathrm{s}^{-1}\right]$ & $3 \times 10^{7} M_{\odot}$ \\
Hydrogen Mass (disc and bridge $M_{H I},\left[F_{H I}=4.0 \mathrm{Jy} \mathrm{km} \mathrm{s}^{-1}\right]$ & $2 \times 10^{8} M_{\odot}$ \\
Face-on gas density $N_{H I},\left[M_{H I} / \pi m_{H} R^{2}\right]$ & $3 \times 10^{19} \mathrm{~cm}^{-2}$ \\
Luminosity limit $L_{B},\left[\mu_{B}>32.8 \mathrm{mag}^{-2} \operatorname{arcsec}^{-2}\right]$ & $<1.9 \times 10^{5} L_{\odot}$ \\
Total Mass to Blue Light Ratio $M_{d y n} / L_{B}$ & $>10^{6} M_{\odot} / L_{\odot}$
\end{tabular}

[assuming a disk 0.5 arcmin $\times 3$ arcmin]

\section{Modelling the Westerbork data}

Previous discussions of the peculiar one armed spiral galaxy NGC4254 have been presented by Iye et al., (1982), Phookun et al., (1993) and Vollmer et al., (2005). In the first of these papers Iye et al. conclude that the most likely cause of the single prominent arm is the growth of a gravitational instability. In the second Phookun et al. argue that the morphological appearance of NGC4254 is due to an instability initiated by infalling HI clouds. In the third paper Vollmer et al. argue that NGC4254 is the result of a rapid and close encounter (along with some ram pressure stripping) with another massive galaxy which they tentatively identify as NGC4262. None of these interpretations can explain the subsequent $21 \mathrm{~cm}$ observations that we have obtained at Jodrell bank, Arecibo and Westerbork. Below we will show the results of a numerical model of an interaction of a dark galaxy with NGC4254 (see also Duc these proceedings).

The numerical code we use is a Treecode-SPH algorithm described by Hernquist and Katz (1989) modified and validated by Williams (1998). Additional details can be found in Williams and Nelson (2001). We model the gas as a compressible fluid, with the addition of gravitational forces and it is kept in an isothermal state at $\approx 10^{4} \mathrm{~K}$. The stars and dark matter obey the collisionless Boltzmann equation. We use a gravity softening length of 0.05 simulation units which corresponds to $0.6 \mathrm{kpc}$.

The simulated initial properties of NGC4254 and VIRGOHI21 are set as follows. The HI mass of NGC4254 is $5.5 \times 10^{9} \mathrm{M}_{\odot}$. For a B band apparent magnitude of 10.44 , a distance of $17 \mathrm{Mpc}$ (the distance of the centroid of the Virgo cluster) and $\mathrm{M}_{\odot}^{B}=5.4$ we have an approximate stellar mass of $2.4 \times 10^{10} \mathrm{M}_{\odot}$. The dynamical mass as inferred from the last point on the rotation curve of Phookum et al., (1993) is $1.0 \times 10^{11} \mathrm{M}_{\odot}$. This leads to a baryonic disc to dark matter halo mass ratio of $m_{d}=0.3$. As this last measured point is still on the flat part of the rotation curve this must be a lower limit to the total mass. Mo et al., (1998) conclude that $m_{d} \leqslant 0.05$ for typical spiral galaxies so we have set the dark matter halo mass to be $6.0 \times 10^{11} \mathrm{M}_{\odot}$ i.e. $m_{d}=0.05$. The dark matter is distributed within an NFW halo in which we embed the gas and stellar discs using the prescription of Mo et al., (1998). With a NFW concentration index of $\mathrm{c}=14$ and a spin parameter of $\lambda=0.058$ the Mo et al. prescription gives an exponential disc scale length for gas and stars of $4 \mathrm{kpc}$ and a rotational velocity of $150 \mathrm{~km} \mathrm{~s}^{-1}$ at three disc scale lengths. This corresponds to an observed scale length of $3.7 \mathrm{kpc}$ and an asymptotic velocity of $150 \mathrm{~km} \mathrm{~s}^{-1}$ (Phookum et al., 1993). Initially particles are laid down at random such that they follow the NFW density distribution (dark matter) or an exponential disc (stars and gas). The particle initial velocities are derived from the formulae given in Widrow (2000) for the dark matter and Mo et al., (1998) for the rotationally supported disc. Initially the gas and star particles are positioned in the plane $(\mathrm{z}=0)$, but as they 
a)

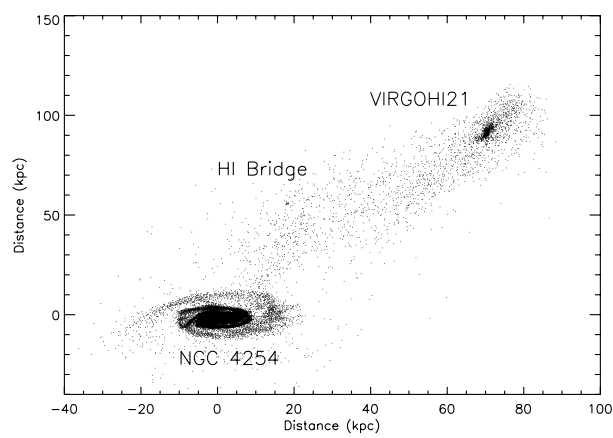

b)

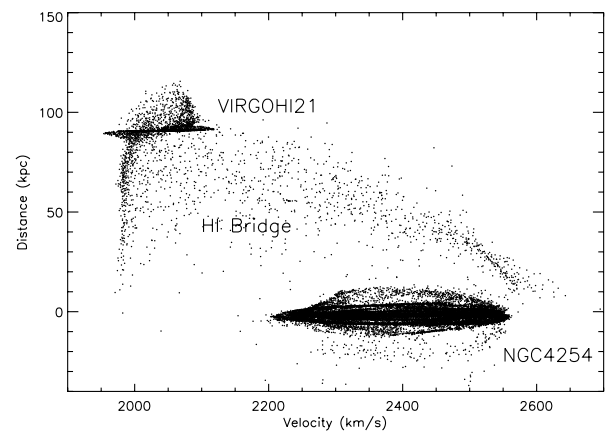

Figure 4. The result of the simulation after 2.25 Gyr. a) - The x-y position of the gas particles. b)) - The $y$ position of the gas particles plotted against the line of sight $(z)$ velocity.

are also given a small velocity dispersion of $10 \mathrm{~km} \mathrm{~s}^{-1}$ the disc initially thickens before settling into its equilibrium position.

VIRGOHI21 is again modelled as a dark matter NFW halo with a gas disc (no stars). The dark halo has a mass of $2.0 \times 10^{10} \mathrm{M}_{\odot}$ (Table 2 ) in which we embed a gas disc of mass $1.0 \times 10^{9} \mathrm{M}_{\odot}$ - this again assumes that initially VIRGOHI21 had $m_{d}=0.05$. As we will see below during the interaction VIRGOHI21 loses a large fraction of its gas mass and much of this is at column densities below the detection threshold of the Westerbork data. Following Mo et al., we embed within the dark halo an exponential gas disc which for this sized halo has a scale length of $4.5 \mathrm{kpc}$ and a rotation velocity at three scale lengths of $53 \mathrm{~km} \mathrm{~s}^{-1}$ (the velocity taken from the observed HI velocity width (after the interaction) is between 75 and $100 \mathrm{~km} \mathrm{~s}^{-1}$ depending on the exact point at which the HI bridge ends and the gas disc begins). With these parameters the central gas column density is $\approx 10^{21}$ which means that the rest of the disc is below the threshold normally assumed for star formation to occur. To achieve this relatively high velocity and low column density we have set the NFW concentration parameter to $\mathrm{c}=14$ and the spin parameter to $\lambda=0.2$. Initial particle positions and velocities are assigned as described above with the exception that the initial line of sight velocity dispersion is reduced to $3 \mathrm{~km} \mathrm{~s}^{-1}$ (so that it is the same fraction of the rotation velocity as that assumed for NGC4254). We now set the initial positions, orientations and relative velocities of NGC4254 and VIRGOHI21 to see if we can reproduce the observations presented in Fig 3. This is a large parameter space to explore, but it is not our intention to try and define exactly the parameters of the interaction only to counter criticism that the observations are inconsistent with an interaction with a dark galaxy but are instead some form of tidal stream.

Quantitatively we believe that our simulation is a reasonably good interpretation of the observations (Fig. 4) as our main intention was to model the ' $Z$ ' like structure seen in the position velocity plot (Fig. 3b). VIRGOHI21 can be seen to be in the correct position in relation to NGC4254 (For $D_{\text {Virgo }}=17 \mathrm{Mpc}$ VIRGOHI21 is at a projected distance of 90 and $70 \mathrm{kpc}$ in the declination and right ascension directions respectively) and there is clearly a HI bridge that links the two together, though it appears much more diffuse than that shown in Fig 3 . There is considerable debris over a large area with VIRGOHI21 being linked to NGC4254 via the bridge. There is also a long drawn out arm behind VIRGOHI21 in the plane of the sky (Fig. 5). NGC4254 has one prominent spiral arm as observed. The HI sizes of the two objects are comparable to those observed. We have carried out another simulation in which VIRGOHI21 is modelled as just a dark halo (no gas and stars) and no gas is drawn out from NGC4254 into a bridge, so all of the 


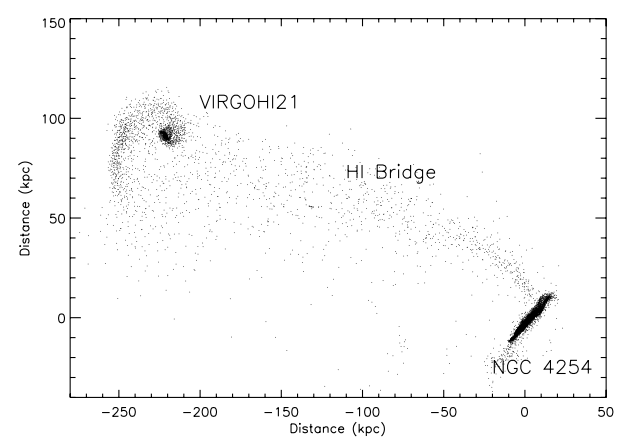

Figure 5. The result of the simulation after $2.25 \mathrm{Gyr}$ - the z-y position of the gas particles.

debris gas seen in Fig. 4a originates from VIRGOHI21. In Fig. 4b we show the y position velocity plot where the velocity is that projected along the line of sight. NGC4254 and VIRGOHI21 are separated by about the correct velocity. Again there is considerable debris, though a number of interesting features have developed. The apparent gas disc of VIRGOHI21 is now much broader in velocity than at the start of the simulation and corresponds very well with that observed. There is gas to the north of VIRGOHI21 though it does not extend very far and is associated with the drawn out arm. There is a strong feature that extends from the disc and at right angles to it that is very similar to the bridge seen in the observational data, though it does not point so clearly at NGC4254, this is again due to the material in the drawn out arm.

In Fig. 6 we attempt to present the simulations in a way that corresponds more closely with the observations shown in Fig 3. Fig. 6a shows a contour map produced by summing the gas particles over an area the size of the resolution element $\left(99^{\prime \prime} \times 30^{\prime \prime}\right)$. The contour levels are exactly the same as those set in Fig 3. The contours show an extended bridge pointing from VIRGOHI21 towards NGC4254 as do the observations. In Fig 6b we again plot the y position of particles against the line of sight velocity only this time we only plot particles that lie in regions where the column density is above the detection limit of $2.5 \times 10^{19} \mathrm{~cm}^{2}$. Now there is far less debris and the shape and positions of the changes in velocity closely resemble the ' $\mathrm{Z}$ ' in the observational data shown in Fig 3 . The distance scale marked on the axes of Fig 5 correspond to the angular scale shown in Fig.3 assuming a distance of $17 \mathrm{Mpc}$.

Measuring directly from the simulation we derive a gas mass of $\approx 6 \times 10^{8} M_{\odot}$ for gas at column densities above the detection threshold. This is compared to a total observed gas mass of $\approx 2 \times 10^{8} M_{\odot}$. We calculate from the simulation a dynamical mass $\left(\frac{r(\Delta v / 2)^{2}}{G}\right)$ of about $3 \times 10^{10} M_{\odot}$ compared to the dark matter halo mass of $2 \times 10^{10} M_{\odot}$.

\section{Conclusions}

In this paper we have demonstrated that there is the possibility of dark galaxies forming with atomic hydrogen and no stars. These galaxies are predominately of low mass and have narrow velocity widths, but they should be detectable by the new, more sensitive, blind HI surveys. The model can be used to predict how many dark galaxies might be expected to be found in these new surveys. Given the problems with finding optical counterparts of HI sources we believe that there is still the possibility that the HIPASS catalogue (HICAT) contains some dark galaxies. We have described a simulation that 

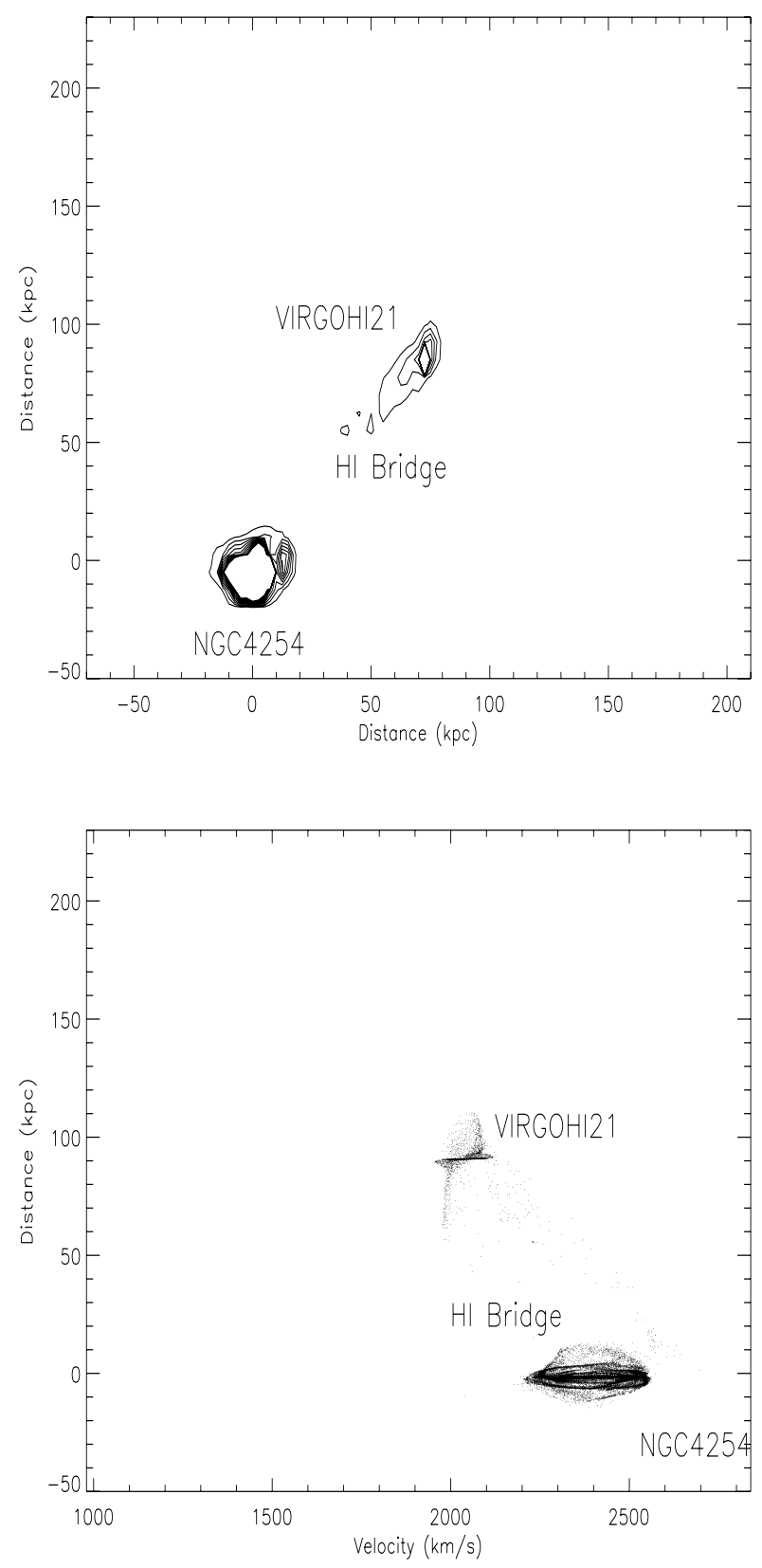

Figure 6. The result of the simulation after 2.25 Gyr. Top - The gas column density with contours set at the same level as those shown in Fig. 3a. Column densities have been derived from the number of gas particles in each $99^{\prime \prime} \times 30^{\prime \prime}$ resolution element. Bottom - The $y$ position of gas particles plotted against the line of sight $(z)$ velocity only for particles where the gas column density is above $2.5 \times 10^{19} \mathrm{~cm}^{-2}$. Both figures can be compared with Fig 3 as the distance scale correspond to the same angular size if the galaxies are at a distance of $17 \mathrm{Mpc}$. 
models the interaction of NGC4254 with a dark matter halo and gas disc. This shows quantitative agreement with observations of VIRGOHI21.

\section{References}

Dalcanton, J., Spergel, D., \& Summers, F., ApJ, 482, 659

Davies, J., et al., 2004, MNRAS, 349, 922

Davies, J. I, Disney, M. J., Minchin, R. F., Auld, R., \& Smith, R., 2006, MNRAS, 368, 1479

Doyle, et al., 2005, MNRAS, 361, 34

Efstathiou, G., Lake G., \& Negroponte, J., 1982, MNRAS, 199, 1069

Hernquist, L., \& Katz N., 1989, ApJS, 70, 419

Iye, M., Okamura, S., \& Watanabe, M., 1982, ApJ, 256, 103

Koribalski, B. et al., 2004, AJ, 128, 16

Maloney, P., 1993, ApJ, 414, 41

Minchin, R., et al., 2005, ApJ, 622, L21

Minchin, R. et al., 2007, ApJ, in press

Mo, H., Mao S., \& White, S., 1998, MNRAS, 295, 319

Navarro, J., Frenk, C., \& White, S., 1995, MNRAS, 275, 56

Navarro, J., Frenk, C., \& White, S., 1996, ApJ, 462, 563

Phookun, B., Vogel, S., \& Mundy, L., 1993, ApJ, 418, 113

Oosterloo, T., \& van Gorkom, J., 2005, $A A, 437,190$

Phookun B., Vogel S. N., \& Mundy L. G., 1993, ApJ, 418, 113

Schaye, J., 2004, ApJ, 609, 667

Taylor, E. N., \& Webster, R. L., 2005, ApJ, 634, 1067

Verde, L., Oh S. P., \& Jimenez, R., 2002, MNRAS, 336, 541

Vollmer, B., Huchtmeier, W., \& van Driel, W., 2005, AA, 439, 921

Warren, M., Quinn, P., Salmon, J., \& Zurek, W., 1992, ApJ, 399, 405

Williams, P., 1998, Ph.D. Thesis, Cardiff University

Williams, P., \& Nelson, A., 2001,AA, 374, 839

Wilson, G., Kaiser, N., \& Luppino, G., ApJ, 2001, 556, 601

Widrow, L.,2000, ApJ, 131, 39 